\title{
Expression of syndecan-1 and cathepsins D and K in advanced esophageal squamous cell carcinoma
}

\author{
Justyna Szumilo', Franciszek Burdan², Krzysztof Zinkiewicz³ ${ }^{3}$ Jaroslaw Dudka ${ }^{1}$, \\ Robert Klepacz ${ }^{1}$, Andrzej Dabrowski ${ }^{3}$, Elzbieta Korobowicz ${ }^{1}$
}

${ }^{1}$ Department of Clinical Pathomorphology, ${ }^{2}$ Experimental Teratology Unit of Human Anatomy Department, 32nd Department of General Surgery, Medical University of Lublin, Lublin, Poland

\begin{abstract}
The key features of malignant neoplasms are their local invasiveness and metastatic potential. Syndecan- 1 - integral membrane heparan sulfate proteoglycan and cathepsins $\mathrm{D}$ and $\mathrm{K}$ - lysosomal proteases are important factors influencing different aspects of these processes. The study was undertaken to determine their expression in esophageal squamous cell carcinoma, and analyze relationship to selected clinicopathological features as well as to survival. Formalin-fixed, paraffin-embedded sections from 39 advanced esophageal squamous cell carcinoma were used for immunohistochemical staining. The epithelial and stromal staining were evaluated separately and compared to conventional clinicopathological features and one-year survival. Positive epithelial immunostaining for syndecan-1, cathepsin D and $\mathrm{K}$ were observed in $82.05 \%, 56.41 \%$ and $30.77 \%$ of tumors, respectively. However, stromal staining was noted in $51.28 \%, 51.28 \%$ and $46.15 \%$ ones, respectively. Epithelial syndecan-1-positive cases were significantly more frequent in well- and moderately differentiated carcinomas. Stromal cathepsin D expression predominated in tumors with infiltrative growth pattern. However, there were no statistically significant differences between any marker-positive and -negative groups with respect to other clinicopathological features studied. The only factors significantly influencing one-year survival were epithelial cathepsin D staining and distant metastasis. In a group of patients who survived one year post surgery, the percentage of cases with negative epithelial cathepsin D staining and without features of distant metastasis were higher. The results may suggest a relationship between syndecan-1 and cathepsins $\mathrm{D}$ and $\mathrm{K}$ with growth and invasiveness of esophageal squamous cell carcinoma, but such thesis requires further study on a larger and more heterogeneous population.
\end{abstract}

Key words: squamous cell carcinoma, esophagus, syndecan-1, cathepsin D, cathepsin K, immunohistochemistry

\section{Introduction}

Esophageal squamous cell carcinoma (ESCC) is relatively rare in Poland - it was the thirteenth most common tumor in males and the thirty third in females according to registered incidence of malignances in 2002 [1]. However, both in Poland and in rest of the world, it is regarded as one of the most lethal neoplasm. At present, a 5-year survival for the whole group of patients with this cancer is about $15 \%$ despite great progress in early detection of precursor lesions and early cancers, especially in high-risk populations, along with application of better methods of therapy (advances in surgical and endoscopic techniques, neoadjuvant chemo- and/or radiotherapy, etc.). The

Correspondence: J. Szumilo MD, PhD, Department of Clinical Pathomorphology, Medical University of Lublin, 8 Jaczewskiego Str., PL-20-059, Lublin, Poland; tel. (+4881) 7187325, fax. (+4881) 7187324, e-mail: jszumilo@wp.pl poor prognosis is mostly the result of advanced stage at presentation, which excludes over $50 \%$ of patients from curative treatment [2]. An aggressive clinical behavior of ESCC is a consequence of its high local invasiveness and metastatic potential [3]. These processes are complex but in a simplistic picture, are involved in detachment of neoplastic cells from the primary site, penetration of extracellular matrix (ECM), followed by entrance into the lumen of vessels, migration with lymph or blood, extravasation into distant organs, and growth with formation of secondary tumors [4]. In fact, each step of this cascade of events depends on a variety of cell-cell or cell-matrix interactions mediated by major families of cell adhesion molecules (CAMs) i.e., cadherins, integrins, immunoglobulins, and selectins, however, some others macromolecules like syndecans are also engaged [4,5].

Syndecans are a four-member family of integral membrane heparan sulfate proteoglycans. They are 
ubiquitous molecules and individual cell types or tissues are characterized by distinct pattern of syndecans expression [5]. Due to the localisation and structure of syndecans they interact with many macromolecules including growth factors (e.g., fibroblast growth factor - FGF, hepatocyte growth factor - HGF, vascular endothelial growth factor - VEGF), ECM components (e.g., fibronectin, vitronectin, laminins and the fibrillar collagens) or cell-cell adhesion molecules (e.g., Lselectin, platelet-endothelial cell adhesion molecules PECAM-1, Mac-1) and via CASK (calcium/calmodulin-dependent serine protein kinase), $\alpha$-actinin and family of actin-binding proteins containing FERM domain with actin cytoskeleton. In this fashion, they may influence a variety of cellular functions like: adhesion, cell signaling, growth, differentiation and motility both, in physiologic and pathologic conditions $[5,6]$. Recent changes in the syndecans' expression, including the best characterized syndecan-1 (CD138), revealed in many precursor lesions and carcinomas are believed to contribute to their progression [6].

The other important aspect of tumor spread is destruction of ECM as a consequence of secretion of different proteolytic enzymes i.e., cysteine-, serineand aspartic proteases, as well as matrix metalloproteases, by tumor or stromal cells. Enzymes breakdown components of ECM including basement membrane that allows for the formation of passageways for the migration of neoplastic cells [7]. Cathepsins D (EC 3.4.23.5) and $\mathrm{K}$ (EC 3.4.22.38) are lysosomal proteases that belong to different classes of endopeptydases, i.e., aspartic and cysteine ones respectively $[8,9]$.

Cathepsin D, detected in most human cells, plays an important role in the intracellular catabolism of proteins, direct degradation of ECM components, the activation of precursor forms of some proteases, and the inactivation of their inhibitors. It may stimulate epithelial cell proliferation by binding insulin-like growth factor receptor (IGFR) and by activation/liberation of other growth factors from ECM. Cathepsin D is also associated with p53-dependent apoptosis, angiogenesis, and attenuation of anti-tumor immune response [7,8]. The enzyme is now regarded as an important factor promoting tumor growth and spread in many malignances [7].

On the contrary, cathepsin $\mathrm{K}$ is characterised by strong collagenolytic and elastinolytic activities [9]. Its expression was revealed in osteoclasts participating in bone and cartilage physiological remodelling. Furthermore, the enzyme was also detected in some epithelial cells e.g., of breast, thyroid gland and lung, where it is engaged is their normal development and function including extracellular proteolysis of e.g., thyreoglobulin or ECM turnover [9-11]. Nowadays, cathepsin K is considered as an important factor in pathogenesis of many inflammatory disorders and neoplasms, not solely of the skeletal system $[9,12,13]$.
Since the role of syndecan-1 and cathepsins D and $\mathrm{K}$ in esophageal ESCC remains unclear, the aim of the present study was to determine the immunoexpression of these proteins and to analyse their relationship to the selected clinicopathological features and survival.

\section{Materials and methods}

Patients characteristic. The study comprised 39 patients with advanced squamous cell carcinoma of the middle thoracic part of the esophagus. All patients were treated by surgery alone at the 2nd Department of General Surgery, Medical University of Lublin, Poland from March 1995 to February 2001. Transthoracic subtotal esophagectomy with 3-D lymph nodes dissection was performed in 32 patients $(82.05 \%)$ and transhiatal esophagectomy without thoracotomy in remaining 7 cases $(17.94 \%)$. The study group consisted of 36 men $(92.31 \%)$ and 3 women $(7.69 \%)$ of ages, ranging from 36 to 75 years (mean 57.74; median 57). The follow-up time ranged from 3 to 68 months (mean 16.13; median 12.0). All the patients deceased by the end of December 2003. Analysis of factors related to survival was limited to one-year observation, due to the overall short survival time in the study group. Ten patients were excluded from this analysis since they died within 30 days after the surgery.

Pathological study. The surgical specimens were fixed in $10 \%$ buffered formalin. The sections were taken according to standard protocol (3-8 sections of the tumor, 2 sections of normal-appearing esophageal wall, sections of proximal and distal resection margins, and sections of all resected lymph nodes $-2-75 /$ case). The sections were routinely processed, embedded in paraffin blocks, and stained with hematoxylin and eosin. Evaluation of pathologic stage of the disease was based on the TNM system and the grade of tumor differentiation on the World Health Organization's criteria [14]. Tumor growth pattern were assessed as expansive (well-circumscribed, pushing border) or infiltrative (indistinct, irregular border). Presence of lymphatic/vascular invasion was defined as tumor cells located within distinct endothelial-lined spaces and partly attached to the lining (no accessory techniques of visualization were applied) [15]. Prominent desmoplasia was defined as desmoplastic stroma compassing $>50 \%$ of tumor area. Distinct cuff of lymphocytes in direct vicinity of advancing edges of the tumor was regarded as prominent peritumoral lymphocytic response. Furthermore, the presence of perineural invasion was also noted.

Immunohistochemical study. An immunohistochemical study was performed on $3 \mu \mathrm{m}$ sections from one, representative paraffin block from each primary tumor. For syndecan-1 and cathepsin D the antigen retrieval technique was applied and slices were pretreated in citrate buffer $(0.01 \mathrm{M}, \mathrm{pH} 6.0)$ in a microwave oven $(2 \mathrm{x}$ for $5 \mathrm{~min}$ at $750 \mathrm{~W}$ ). For all antigens the endogenous peroxidase activity was blocked by $3 \%$ hydrogen peroxide solution for 20 minutes at room temperature. To block non-specific binding sites normal goat or rabbit serum was applied for 10 minutes at room temperature for syndecan- 1 and cathepsin $\mathrm{D}$, respectively. This procedure was omitted for the cathepsin $\mathrm{K}$ reaction. In the next step, the sections were incubated with the following primary monoclonal mouse anti-human antibodies against: syndecan-1 (CD138) (clone B-B4, Serotec, UK; dilution 1:100 for $30 \mathrm{~min}$ ), cathepsin D (clone C5, Novocastra, UK; dilution 1:100 for 60 min), and cathepsin K (clone CK4, Novocastra; dilution 1:80 for $60 \mathrm{~min}$ ) at room temperature. This was followed by incubation with appropriate biotynylated secondary antibodies for $30 \mathrm{~min}$ and than streptavidin-biotin complex (LSAB2/HRP; DakoCytomation, Denmark) or avidin-biotin complex (ABComplex/HRP; Novocastra) for $30 \mathrm{~min}$ at room temperature, for syndecan-1 and cathepsin $\mathrm{D}$, respectively. For cathepsin K, DakoEnvision+TM/HRP kit 
(DakoCytomation) was applied in the same conditions. After each step, slides were washed with TBS. The specific immune reactions were visualized by 3',3-diaminobenzidine tetrahydrochloride (DAB) (DakoCytomation). Sections were then counterstained with Mayer's haematoxylin. Slides treated in the same way, but with omission of primary antibodies, were used as negative controls. The following tissue samples were applied as a positive control: vaginal part of uterine cervix for syndecan-1, ductal breast carcinoma - known to be strongly cathepsin D positive, and osteochondroma for cathepsin $\mathrm{K}$. Immunohistochemical reaction was evaluated separately in tumor and stroma by light microscope (Olympus BX45; Japan) equipped with the eye grid. The percentage of positively-stained cells of each category was calculated in approximately 2000 cells of the some type in randomly selected areas using objective $\mathrm{x} 40$ and included the following categories: no positive stained cells or less then $10 \%, 10-50 \%$ of positively-stained cells and more than $50 \%$ of positively-stained cells. For statistical analysis a case was classified as positive if at least $10 \%$ of the cells were positively-stained. All slides were examined independently by two experienced pathologists (J.S., E.K.) without knowledge of the patient's outcome. In cases of disagreement, the slides were reviewed with a double-headed microscope to achieve a consensus.

Statistical analysis. Statistical differences between variables were evaluated using Fischer exact test. Kenndall Tau test was applied for assessment of correlation between variables. Survival rates were calculated using Gehan and log-rank test. P values less than 0.05 were considered significant. Data was analysed by STATISTICA 5.0 on an IBM compatible computer.

\section{Results}

Normal stratified squamous epithelium of the esophagus revealed strong positive membrane syndecan-1 immunostaining of parabasal and intermediate layers that decreased in more superficial layers. The staining was weak on the apico-lateral aspects of basal layer cells, and absent on the basal aspect. Esophageal glands showed weak cytoplasmic staining. A strong reaction was noted in plasma cells. In 3 cases, in which in situ carcinoma was present at the edges of invasive ESCC, syndecan-1 expression was markedly reduced when compared to normal epithelium and the staining was stronger in superficial layers. $32(82.05 \%)$ of the 39 ESCCs were syndecan-1-positive (Fig.1A-B). The staining pattern was predominantly membranous in $56.25 \%$ of the cases and mixed membranous and diffuse cytoplasmic in $43.75 \%$. In better-differentiated areas of tumors, cancer cells recapitulated zonal expression of syndecan-1 typical of normal squamous epithelium. Syndecan-1-positive cases were significantly $(p<0.05)$ more frequent among well and moderately differentiated ESCCs. There were no statistically significant association between epithelial syndecan-1 expression and other clinicopathological findings (Table 1). Syndecan-1 stromal reactivity was seen in 20 cases $(51.28 \%)$ (Fig. 1A-B) and it was stronger among cancer foci and in close vicinity to advancing tumor margins. A positive stromal reaction correlated exclusively with syndecan-1 positive cancer cases $(\tau=0.4799)$ and distant metastasis $(\tau=0.4543)$.
Normal esophageal squamous epithelium and in situ carcinoma were cathepsin D-negative, but the glands were weakly positive. Cathepsin D immunoreactivity was revealed in 22 cases of ESCCs (56.41\%) (Fig. 1C-D). In $72.72 \%$ of the cases, positive cancer cells were located in the most external parts of tumor foci. Observations in the remaining samples showed a homogenous staining of a majority of tumor cells (Fig. 1C). No statistically significant differences were noted between cancer cathepsin D-positive and -negative cases and all the studied clinicopathological findings (Table. 1). However, significant correlations were revealed between epithelial cathepsin D-positive staining and stromal cathepsin D- and epithelial cathepsin K-positive staining ( $\tau=0.5915$ and $\tau=0.2499$, respectively), distant metastasis $(\tau=0.2315)$, and prominent desmoplasia $(\tau=0.2315)$. Distinct stromal positive cathepsin D staining was seen in 20 cases $(51.28 \%)$ (Fig. 1C-D). An especially strong granular cytoplasmic staining was noted in macrophages that infiltrated the cancer area. Distribution of strong positive fibroblasts was similar to those of syndecan-1 samples. Stromal cathepsin D expression was statistically more frequent in tumors with as infiltrative, as opposed to expansive, growth pattern $(\mathrm{p}<0.05)$ (Table 1$)$. Positive stromal cathepsin D staining statistically correlated with a positive cathepsin $\mathrm{D}$ cancer staining $(\tau=0.5915)$, a distant metastasis $(\tau=0.2734)$, and an infiltrative growth pattern $(\tau=0.3374)$.

Normal squamous epithelium, esophageal glands, and in situ carcinoma were cathepsin K-negative. Cathepsin K immunoreactivity was noted in only 12 studied cases of ESCCs (30.77\%) (Fig. 1E). The reaction was confined to relatively sparse cancer cells located externally in tumor foci. Positive cathepsin K epithelial staining correlated with positive cathepsin $\mathrm{K}$ stromal $(\tau=0.2743)$ and cathepsin D epithelial $(\tau=0.2499)$ staining, prominent desmoplasia $(\tau=0.3316)$, and an expansive growth pattern $(\tau=0.2642)$. Stromal cathepsin K staining was found in 18 cases $(46.15 \%)$ (Fig. 1E-F). Strong granular cytoplasmic staining was observed in macrophages, especially in osteoclast-like multinucleated giant cells, seen mainly at the edges of keratinized cancer foci (Fig. 1F). Relative to cathepsin D, a smaller number of macrophages exhibited cathepsin $\mathrm{K}$ reactivity. The intensity of fibroblast staining was weak. Similar to cancer immunoreactivity, no statistically significant differences between stromal cathepsin K-positive and -negative cases and all the clinicopathological findings were noted (Table 1). Cathepsin K stromal immunoexpression significantly correlated with positive epithelial cathepsin $\mathrm{K}$ staining $(\tau=0.2743)$ and a better differentiation of tumors $(\tau=0.3090)$.

The only factors that significantly influenced oneyear survival in the study group were cancer cathepsin D 

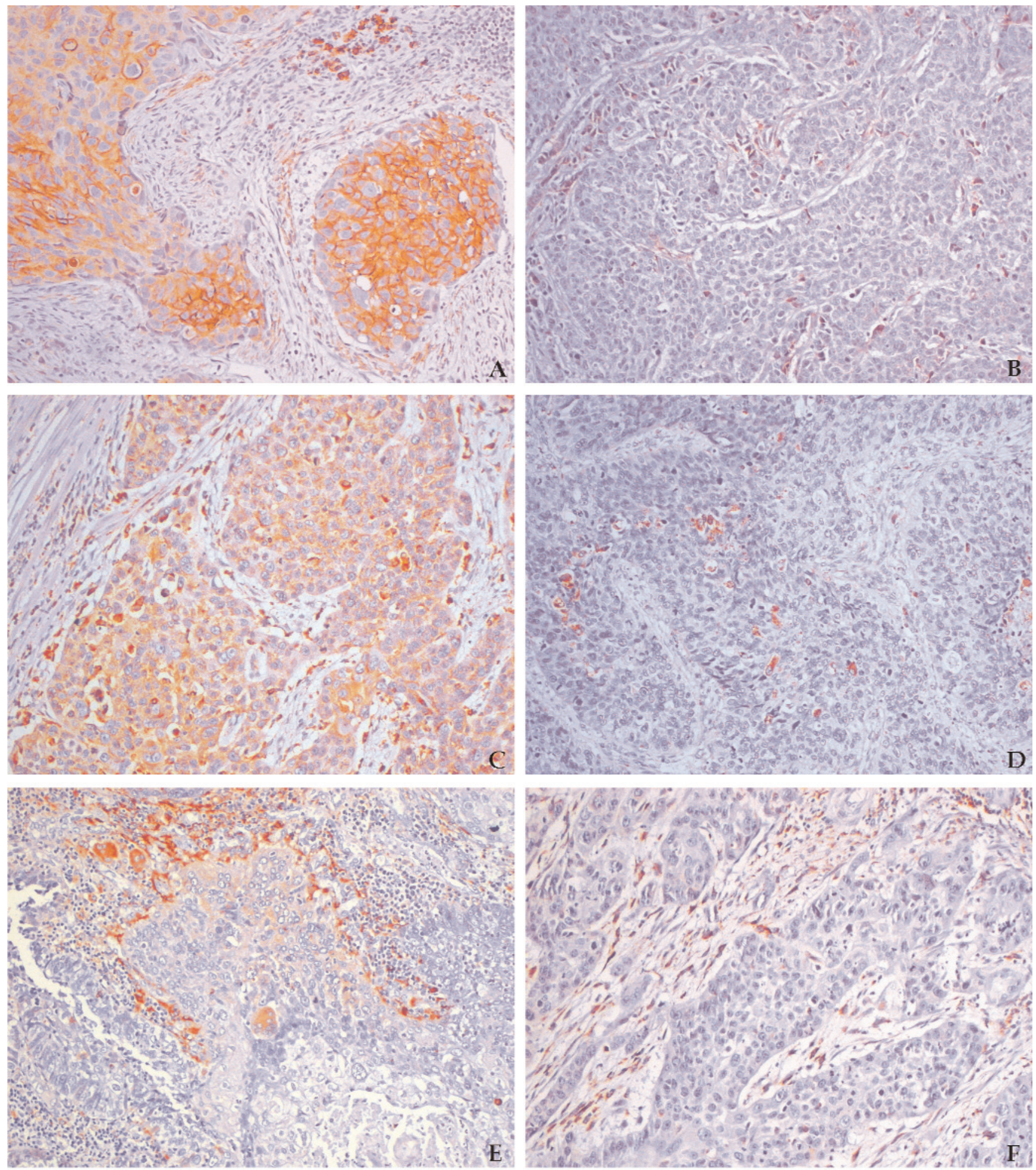

Fig. 1. Positive (A, C, E) and negative (B, D, F) epithelial immunohistochemical reaction for syndecan-1 $(\mathbf{A}, \mathbf{B})$, cathepsin D $(\mathbf{C}, \mathbf{D})$ and cathepsin K $(\mathbf{E}, \mathbf{F})$, respectively. Positive stromal reactions for all antigens studied (original magnification $\times 10$ ).

staining and distant metastasis $(\mathrm{p}<0.05)$. In patients with ESCC, who survive one year after the surgery, the percentage of cases with negative cancer cathepsin D staining (Fig. 2) and without features of distant metastasis, was higher.

\section{Discussion}

The current study demonstrated immunoexpression of three molecules that are probably involved in tumor growth and dissemination in advanced ESCCs. 
Table 1. Relation between immunoexpressions of syndecan-1 and cathepsins D and K and selected clinicopathological findings in patients with esophageal squamous cell carcinoma

\begin{tabular}{|c|c|c|c|c|c|c|c|c|c|c|c|c|}
\hline \multirow[b]{2}{*}{ Variable } & \multicolumn{4}{|c|}{ Syndecan-1 } & \multicolumn{4}{|c|}{ Cathepsin D } & \multicolumn{4}{|c|}{ Cathepsin K } \\
\hline & $\begin{array}{c}\text { ESCC } \\
(+) \\
\mathrm{n}(\%)\end{array}$ & $\begin{array}{c}\text { ESCC } \\
(-) \\
\mathrm{n}(\%) \\
\end{array}$ & \begin{tabular}{c|}
$\begin{array}{c}\text { Stroma } \\
(+) \\
\mathrm{n}(\%)\end{array}$ \\
\end{tabular} & $\begin{array}{c}\text { Stroma } \\
(-) \\
\mathrm{n}(\%)\end{array}$ & $\begin{array}{c}\mathrm{FSCC} \\
(+) \\
\mathrm{n}(\%)\end{array}$ & $\begin{array}{c}\mathrm{ESCC} \\
(-) \\
\mathrm{n}(\%)\end{array}$ & $\begin{array}{c}\text { Stroma } \\
(+) \\
\mathrm{n}(\%)\end{array}$ & $\begin{array}{c}\text { Stroma } \\
(-) \\
\mathrm{n}(\%) \\
\end{array}$ & \begin{tabular}{c|}
$\mathrm{ESCC}$ \\
$(+)$ \\
$\mathrm{n}(\%)$ \\
\end{tabular} & \begin{tabular}{|c|} 
ESCC \\
$(-)$ \\
$\mathrm{n}(\%)$ \\
\end{tabular} & \begin{tabular}{c|}
$\begin{array}{c}\text { Stroma } \\
(+) \\
\mathrm{n}(\%)\end{array}$ \\
\end{tabular} & $\begin{array}{c}\text { Stroma } \\
(-) \\
\mathrm{n}(\%)\end{array}$ \\
\hline$\geq 6(\mathrm{n}=15)$ & $\begin{array}{c}20 \\
(62.5) \\
12 \\
(37.5)\end{array}$ & $\begin{array}{c}4 \\
(57.14) \\
3 \\
(42.86)\end{array}$ & $\begin{array}{c}13 \\
(65.0) \\
7 \\
(35.0)\end{array}$ & $\begin{array}{c}11 \\
(57.89) \\
8 \\
(42.11)\end{array}$ & $\begin{array}{c}12 \\
(54.55) \\
10 \\
(45.45)\end{array}$ & $\begin{array}{c}12 \\
(70.59) \\
5 \\
(29.41)\end{array}$ & $\begin{array}{c}10 \\
(50.0) \\
10 \\
(50.0)\end{array}$ & $\begin{array}{c}14 \\
(73.68) \\
5 \\
(26.32)\end{array}$ & $\begin{array}{c}5 \\
(41.67) \\
7 \\
(58.33)\end{array}$ & $\begin{array}{c}19 \\
(70.37) \\
8 \\
(29.63)\end{array}$ & $\begin{array}{c}11 \\
(61.11) \\
7 \\
(38.89)\end{array}$ & $\begin{array}{c}13 \\
(61.90) \\
8 \\
(38.10)\end{array}$ \\
\hline $\begin{array}{l}\text { invasion }^{2} \\
\text { pT2 }(n=1) \\
\text { pl3/4 }(n=38)\end{array}$ & $\begin{array}{c}1 \\
(3.13) \\
32 \\
(96.87) \\
\end{array}$ & $\begin{array}{c}0 \\
7 \\
(100.0) \\
\end{array}$ & $\begin{array}{c}0 \\
20 \\
(100.0) \\
\end{array}$ & $\begin{array}{c}1 \\
(5.26) \\
18 \\
(94.74) \\
\end{array}$ & $\begin{array}{c}0 \\
22 \\
(100.0) \\
\end{array}$ & $\begin{array}{c}1 \\
(5.88) \\
16 \\
(94.12)\end{array}$ & $\begin{array}{c}0 \\
20 \\
(100.0)\end{array}$ & $\begin{array}{c}1 \\
(5.26) \\
18 \\
(96.74)\end{array}$ & $\begin{array}{c}0 \\
12 \\
(100.0) \\
\end{array}$ & $\begin{array}{c}1 \\
(3.70) \\
26 \\
(26.30) \\
\end{array}$ & $\begin{array}{c}0 \\
18 \\
(100.0) \\
\end{array}$ & $\begin{array}{c}1 \\
(4.76) \\
20 \\
(95.24)\end{array}$ \\
\hline $\begin{array}{l}\mathrm{pNO}(\mathrm{n}=7) \\
\mathrm{pNl}(\mathrm{n}=32)\end{array}$ & $\begin{array}{c}6 \\
(18.75) \\
26 \\
(81.25) \\
\end{array}$ & $\begin{array}{c}1 \\
(14.29) \\
6 \\
(85.71) \\
\end{array}$ & $\begin{array}{c}4 \\
(20.0) \\
16 \\
(80.0) \\
\end{array}$ & $\begin{array}{c}3 \\
(15.79) \\
16 \\
(84.21) \\
\end{array}$ & $\begin{array}{c}4 \\
(18.18) \\
18 \\
(81.82) \\
\end{array}$ & $\begin{array}{c}3 \\
17.65) \\
14 \\
(82.35) \\
\end{array}$ & $\begin{array}{c}4 \\
20.0) \\
16 \\
(80.0) \\
\end{array}$ & $\begin{array}{c}3 \\
(15.79) \\
16 \\
(84.21) \\
\end{array}$ & $\begin{array}{c}1 \\
(8.33) \\
11 \\
(91.67) \\
\end{array}$ & $\begin{array}{c}6 \\
(22.22) \\
21 \\
(77.78) \\
\end{array}$ & $\begin{array}{c}3 \\
(16.67) \\
15 \\
(83.33) \\
\end{array}$ & $\begin{array}{c}4 \\
(19.05) \\
17 \\
(80.95) \\
\end{array}$ \\
\hline $\begin{array}{l}\mathrm{pM} 0(\mathrm{n}=33) \\
\mathrm{pM} 1(\mathrm{n}=6)\end{array}$ & $\begin{array}{c}26 \\
(81.25) \\
6 \\
(18.75) \\
\end{array}$ & $\begin{array}{c}7 \\
(100.0) \\
0\end{array}$ & $\begin{array}{c}15 \\
(75.0) \\
5 \\
(25.0) \\
\end{array}$ & $\begin{array}{c}18 \\
(94.74) \\
1 \\
(5.26) \\
\end{array}$ & $\begin{array}{c}17 \\
(77.27) \\
5 \\
(22.73) \\
\end{array}$ & $\begin{array}{c}16 \\
(94.12) \\
1 \\
(5.88)\end{array}$ & $\begin{array}{c}15 \\
(75.0) \\
5 \\
(25.0) \\
\end{array}$ & $\begin{array}{c}18 \\
(96.74) \\
1 \\
(5.26) \\
\end{array}$ & $\begin{array}{c}11 \\
(91.67) \\
1 \\
(8.33)\end{array}$ & $\begin{array}{c}22 \\
(81.48) \\
5 \\
(18.52) \\
\end{array}$ & $\begin{array}{c}14 \\
(77.78) \\
4 \\
(22.22) \\
\end{array}$ & $\begin{array}{c}19 \\
(90.48) \\
2 \\
(9.52)\end{array}$ \\
\hline $\begin{array}{l}\text { II }(\mathrm{n}=7) \\
\mathrm{III} / \mathrm{IV}(\mathrm{n}=\end{array}$ & $\begin{array}{c}6 \\
(18.75) \\
26 \\
(81.25) \\
\end{array}$ & $\begin{array}{c}1 \\
(14.29) \\
6 \\
(85.71) \\
\end{array}$ & $\begin{array}{c}4 \\
(20.0) \\
16 \\
(80.0) \\
\end{array}$ & $\begin{array}{c}3 \\
(15.79) \\
16 \\
(84.21)\end{array}$ & $\begin{array}{c}4 \\
(18.18) \\
18 \\
(81.82) \\
\end{array}$ & $\begin{array}{c}3 \\
(17.65) \\
14 \\
(82.35)\end{array}$ & $\begin{array}{c}4 \\
(20.0) \\
16 \\
(80.0)\end{array}$ & $\begin{array}{c}3 \\
(15.79) \\
16 \\
(84.21)\end{array}$ & $\begin{array}{c}1 \\
(8.33) \\
11 \\
(91.67)\end{array}$ & $\begin{array}{c}6 \\
(22.22) \\
21 \\
(77.78)\end{array}$ & $\begin{array}{c}3 \\
(16.67) \\
15 \\
(83.33)\end{array}$ & $\begin{array}{c}4 \\
(19.05) \\
17 \\
(80.95)\end{array}$ \\
\hline $\begin{array}{l}\text { grading } \\
\text { G1 } 1 / 2(\mathrm{n}=24)\end{array}$ & $\begin{array}{c}23^{*} \\
(71.88) \\
9 \\
(28.13)\end{array}$ & $\begin{array}{c}1 \\
(14.29) \\
6 \\
(85.71)\end{array}$ & $\begin{array}{c}14 \\
(70.0) \\
6 \\
(30.0)\end{array}$ & $\begin{array}{c}10 \\
(52.63) \\
9 \\
(47.37)\end{array}$ & $\begin{array}{c}14 \\
(63.64) \\
8 \\
(36.36)\end{array}$ & $\begin{array}{c}10 \\
(55.82) \\
7 \\
(41.18)\end{array}$ & $\begin{array}{c}12 \\
(60.0) \\
8 \\
(40.0)\end{array}$ & $\begin{array}{c}12 \\
(63.16) \\
7 \\
(36.84)\end{array}$ & $\begin{array}{c}8 \\
(66.67) \\
4 \\
(33.33)\end{array}$ & $\begin{array}{c}16 \\
(59.26) \\
11 \\
(40.74)\end{array}$ & $\begin{array}{c}14 \\
(77.78) \\
4 \\
(22.22)\end{array}$ & $\begin{array}{c}10 \\
(47.62) \\
11 \\
(52.38)\end{array}$ \\
\hline $\begin{array}{l}\text { expansive }(n=29) \\
\text { infiltrative }(n=10)\end{array}$ & $\begin{array}{c}24 \\
(75.0) \\
8 \\
(25.0)\end{array}$ & $\begin{array}{c}5 \\
(71.43) \\
2 \\
(28.57)\end{array}$ & \begin{tabular}{c|}
13 \\
$(65.0)$ \\
7 \\
$(35.0)$
\end{tabular} & $\begin{array}{c}16 \\
(84.21) \\
3 \\
(15.79)\end{array}$ & $\begin{array}{c}16 \\
(72.73) \\
6 \\
(27.27)\end{array}$ & $\begin{array}{c}13 \\
(76.47) \\
4 \\
(23.53)\end{array}$ & $\begin{array}{c}12 * \\
(60.0) \\
8 \\
(40.0)\end{array}$ & $\begin{array}{c}17 \\
(89.47) \\
2 \\
(10.53)\end{array}$ & $\begin{array}{c}11 \\
(91.67) \\
1 \\
(8.33)\end{array}$ & $\begin{array}{c}18 \\
(66.67) \\
9 \\
(33.33)\end{array}$ & $\begin{array}{c}12 \\
(66.67) \\
6 \\
(33.33)\end{array}$ & $\begin{array}{c}17 \\
(80.95) \\
4 \\
(19.05)\end{array}$ \\
\hline present $(n=35)$ & $\begin{array}{c}4 \\
(12.50) \\
28 \\
(87.50)\end{array}$ & $\begin{array}{c}0 \\
7 \\
(100.0) \\
\end{array}$ & $\begin{array}{c}2 \\
(10.00) \\
18 \\
(90.00) \\
\end{array}$ & $\begin{array}{c}2 \\
(10.53) \\
17 \\
(89.47)\end{array}$ & $\begin{array}{c}2 \\
(9.09) \\
20 \\
(90.91)\end{array}$ & $\begin{array}{c}2 \\
(11.76) \\
15 \\
(88.24)\end{array}$ & $\begin{array}{c}2 \\
(10.0) \\
18 \\
(90.0)\end{array}$ & $\begin{array}{c}2 \\
(10.53) \\
17 \\
(89.47) \\
\end{array}$ & $\begin{array}{c}2 \\
(16.67) \\
10 \\
(83.33) \\
\end{array}$ & $\begin{array}{c}2 \\
(7.41) \\
25 \\
(92.59) \\
\end{array}$ & $\begin{array}{c}3 \\
(16.67) \\
15 \\
(83.33) \\
\end{array}$ & $\begin{array}{c}1 \\
(4.76) \\
20 \\
(95.24)\end{array}$ \\
\hline $\begin{array}{l}\text { absent }(\mathrm{n}=12) \\
\text { present }(\mathrm{n}=27)\end{array}$ & $\begin{array}{c}9 \\
(28.13) \\
23 \\
(71.88)\end{array}$ & $\begin{array}{c}3 \\
(42.86) \\
4 \\
(57.14)\end{array}$ & $\begin{array}{c}6 \\
(30.0) \\
14 \\
(70.0)\end{array}$ & $\begin{array}{c}6 \\
(31.58) \\
13 \\
(68.42)\end{array}$ & $\begin{array}{c}5 \\
(22.73) \\
17 \\
(77.27)\end{array}$ & $\begin{array}{c}7 \\
(41.18) \\
10 \\
(58.82)\end{array}$ & $\begin{array}{c}5 \\
(25.00) \\
15 \\
(75.00)\end{array}$ & $\begin{array}{c}7 \\
(36.84) \\
12 \\
(63.16)\end{array}$ & $\begin{array}{c}4 \\
(33.33) \\
8 \\
(66.67)\end{array}$ & $\begin{array}{c}8 \\
(29.73) \\
19 \\
(70.37)\end{array}$ & $\begin{array}{c}5 \\
(27.78) \\
13 \\
(72.22)\end{array}$ & $\begin{array}{c}7 \\
(33.33) \\
14 \\
(66.67)\end{array}$ \\
\hline $\begin{array}{l}\text { Prominent desmoplasia } \\
\text { absent }(n=33) \\
\text { present }(n=6)\end{array}$ & $\begin{array}{c}26 \\
(81.25) \\
6 \\
(18.75)\end{array}$ & $\begin{array}{c}7 \\
(100.0) \\
0\end{array}$ & $\begin{array}{c}17 \\
(85.0) \\
3 \\
(15.0)\end{array}$ & $\begin{array}{c}16 \\
(84.21) \\
3 \\
(15.79)\end{array}$ & $\begin{array}{c}17 \\
(77.27) \\
5 \\
(22.73)\end{array}$ & $\begin{array}{c}16 \\
(94.12) \\
1 \\
(5.88)\end{array}$ & $\begin{array}{c}17 \\
(85.0) \\
3 \\
(15.0)\end{array}$ & $\begin{array}{c}16 \\
(84.21) \\
3 \\
(15.79)\end{array}$ & $\begin{array}{c}8 \\
(66.67) \\
4 \\
(33.33)\end{array}$ & $\begin{array}{c}25 \\
(92.59) \\
2 \\
(7.41)\end{array}$ & $\begin{array}{c}16 \\
(88.89) \\
2 \\
(11.11)\end{array}$ & $\begin{array}{c}17 \\
(80.95) \\
4 \\
(19.05)\end{array}$ \\
\hline $\begin{array}{l}\text { Prominent peritumoral } \\
\text { lymphocytic response } \\
\text { absent }(\mathrm{n}=23 \text { ) } \\
\text { present }(\mathrm{n}=16)\end{array}$ & $\begin{array}{c}18 \\
(56.25) \\
14 \\
(43.75)\end{array}$ & $\begin{array}{c}5 \\
(71.43) \\
2 \\
(28.57)\end{array}$ & $\begin{array}{c}12 \\
(60.0) \\
8 \\
(40.0)\end{array}$ & $\begin{array}{c}11 \\
(57.89) \\
8 \\
(42.11)\end{array}$ & $\begin{array}{c}14 \\
(63.64) \\
8 \\
(36.36)\end{array}$ & $\begin{array}{c}9 \\
(52.94) \\
8 \\
(47.06)\end{array}$ & $\begin{array}{c}10 \\
(50.0) \\
10 \\
(50.0)\end{array}$ & $\begin{array}{c}13 \\
(68.42) \\
6 \\
(31.58)\end{array}$ & $\begin{array}{c}9 \\
(75.0) \\
3 \\
(25.0)\end{array}$ & $\begin{array}{c}14 \\
(51.85) \\
13 \\
(48.15)\end{array}$ & $\begin{array}{c}11 \\
(61.11) \\
7 \\
(38.89)\end{array}$ & $\begin{array}{c}12 \\
(57.14) \\
9 \\
(42.86)\end{array}$ \\
\hline
\end{tabular}

(1) median; (2) pT2, pT3, and pT4 spread to muscularis propria, adventitia, and adjacent structures, respectively; (3) pN0, and pN1, absence, and presence of lymph nodes metastasis, respectively; (4) pM0, and pM1, absence, and presence of distant metastasis, respectively; (5) stage II = T2-3 N0 M0 or T1-2 N1 M0, stage III = T3 N1 M0 or T4 N0-1 M0, stage IV = T1-4 N0-1 M1; (6) G1 - well-, G2 - moderately-, and G3 - poorly differentiated ESCC; (*) p<0.05 


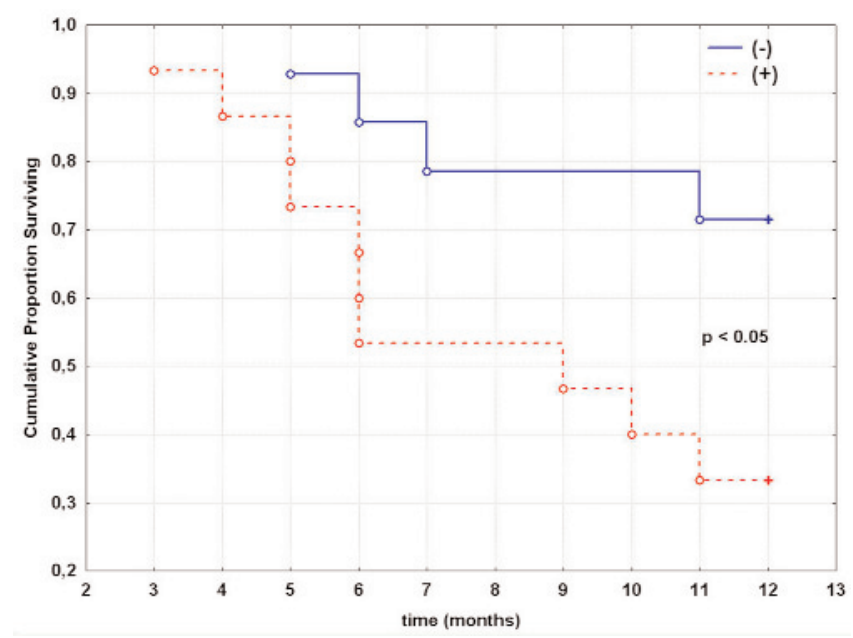

Fig. 2. One-year survival of patients with esophageal squamous cell carcinoma according to epithelial cathepsin D status.

Positive epithelial syndecan-1 immunoexpression was seen in over $80 \%$ of cases. Except positive direct relation with histological grading of cancers, no significant associations between syndecan-1 expression and clinicopathological parameters were revealed. A similar correlation with tumor differentiation was also seen in the squamous cell carcinoma of various locations e.g., of head and neck [16,17], and uterine cervix [18] as well as in adenocarcinomas including that of the stomach [19], large intestine [20], liver (both hepato- and cholangiocellular carcinomas) [21,22], kidney [23] and endometrium [24]. These proved the importance of epithelial syndecan-1 in maintaining differentiating phenotypes, not only in physiological conditions, but in a variety of neoplasms as well. This was supported by experimental findings [5]. It was observed that suppression of syndecan-1 expression on epithelial cells by transfection with antisense cDNA caused profound changes in cell morphology. A flattened, cuboidal shape, typical of epithelial cells, was replaced by an elongated fusiform shape, which was accompanied by the ability to migrate in collagen gels as well as anchorage-independent growth [5]. It should be stressed that in many tumors mentioned above, negative syndecan-1 expression correlated with at least some of the unfavorable markers like more advanced stage, deeper level of infiltration, vascular invasion, lymph node, or distant metastasis [19-25]. An abnormal expression of syndecan-1 was also revealed in precursor lesions. In the present study, a reduction in the expression and its localization in more superficial layers, was noted in in situ carcinoma of the esophagus that was also seen in other organs lined with squamous epithelium [17]. Lost of syndecan-1 expression was observed in colorectal adenomas and endometrial hyperplasia [26]. These findings confirmed the significant role of syndecan-1-status, not only during cancer dissemination, but also in the early, intraepithelial stage as well. It seems that the decrease of syndecan-1 expression in neoplastic cells influenced their growth and morphology and caused a reduction of cell-cell, followed by cell-matrix interactions. This phenomenon precluded and influenced the reduction of another adhesion molecule expression - E-cadherin $[19,26]$. Such changes facilitate cancer cell migration and stromal infiltration, as well as vascular invasion and metastasis. However, enhanced syndecan-1 expression correlated with increased aggressiveness and a poor clinical outcome in breast [27], prostate [28] and nasopharyngeal cancers [29]. This dualistic role of syndecan- 1 in carcinogenesis may be the consequence of a tissue and/or tumor stage-specific function, and reflects multiple functions of the molecule [6].

Contribution of cathepsin D in growth and dissemination of various tumors was extensively studied. Increased cathepsin D immunostaining was detected in some precursor lesions, e.g., in grade III endometrial adenomatous hyperplasia [30] or colorectal adenomas [31]. Some reports revealed increased cathepsin D activity in serum and/or tumor homogenates, especially in malignancies [32]. Strong cathepsin D immunoexpression was found in nasopharyngeal [33], oral [34], gastric [35], colorectal [31], renal [36], breast [37] and ovarian [38] carcinomas. In our study, positive cathepsin D staining in cancer cells was found in slightly more than half of the ESCCs cases. Although no significant differences between cathepsin D-positive and -negative cases with respect to studied clinicopathological features were revealed, the majority of patients who survive one year after the surgery were characterized by cathepsin D negative cancer staining. Epithelial expression correlated with distant metastasis and prominent desmoplasia. These findings may suggest the influence of cathepsin D over ESCCs invasiveness. Despite differences in the examined group, especially as far as the number of cases, staging and outcome are concerned, our results were partly supported by the only report a ESCC by Ikeguchi et al. [39]. Epithelial cathepsin D imunoexpression was noted in $49 \%$ of ESCCs. It was significantly associated with a tumor invasive growth pattern, poor prognosis, and nuclear accumulation of p53 protein, but cathepsin D expression was not recognized as an independent prognostic factor in multivariate survival analysis. Correlating with the majority of our cases, cathepsin D-positive cancer cells were usually present at the advancing margins rather than in the central part of the tumor. Many experimental studies gave evidence of the potential role of cathepsin D in tumor invassivenes and metastatic ability. It was proven that cathepsin D may directly participate in ECM degradation after its release from cells and its autoactivation at low $\mathrm{pH}$, typical of tumor stroma, or indirectly via acti- 
vation of a cascade of other proteases like cathepsin B, $\mathrm{L}$, plasminogen activators, plasmin and matrix metaloproteases [8]. Furthermore, cathepsin D as a mitogen, together with various factors, released from the damaged ECM or from tumor-associated macrophages and other inflammatory cells could directly stimulate cancer growth and angiogenesis [7]. In fact, the positive correlation of epithelial cathepsin D expression with lymph node or distant metastasis, as well as with advanced stage was reported [31-35]. It is worth mentioning however, that in renal cell carcinomas strong cathepsin D imunoexpression was associated with a significantly lower incidence of distant metastasis and an improved long-term survival [36].

According to our knowledge, the current study is the first to describe the immunoexpression of cathep$\sin \mathrm{K}$ in ESCCs. Cathepsin K was found in sparse cancer cells in almost $1 / 3$ of the cases. Its expression correlated with the expansive growth pattern and prominent desmoplasia. It is interesting that epithelial cathepsin $\mathrm{K}$ was detected in malignancies known for their special propensity to bone metastases like cancer of prostate [12], breast [10] and lung [13]. In ESCCs bone metastases are also relatively common and occur in $9-20 \%$ of cases [14]. Based on cathepsin K mRNA and protein expression in prostate cancer cell lines and tissue samples, Brubaker et al. [12] indicated that in primary sites, cathepsin $\mathrm{K}$ may promote cancer dissemination, but in osseous metastasis, it is involved in degradation of ECM. However, in the present study, significant differences in cathepsin $\mathrm{K}$ expression with respect to nodal and distant metastasis were not revealed. It is worth mentioning that cathepsin $\mathrm{K}$ is characterised by strong and unique proteolytic activity and is able to breakdown bone matrix components i.e., type I collagen, osteopontin and osteonectin [9]. It is not surprising that the protease is engaged in bone destruction in many non-epithelial tumours and tumour-like conditions e.g. osteosarcoma [40] or giant cell tumour [41]. The cathepsin released by osteoclasts and tumor cells degrades bone matrix but also may participate in the establishment of micrometastases and lead to the release of factors supporting tumor growth [12].

It is interesting that in the current study stromal expressions of all the macromolecules were a frequent finding, revealed in approximately half of samples. Stromal cathepsin D immunoexpression was statistically more frequent in tumors with an infiltrative growth pattern. Furthermore, syndecan-1 and cathepsin D expression correlated with distant metastasis, but cathepsin $\mathrm{K}$ was associated with better differentiation of tumors. Syndecan-1 was also detected in the stroma of colorectal [20], pancreatic [42], endometrial [24], and head and neck [17] cancers. A few authors reported its association with poor prognosis $[20,42]$.
On the other hand, in invasive breast cancers, positive stromal cathepsin D immunoexpression was common and linked with unfavorable prognostic markers, i.e., advanced tumor stage, lower grade of differentiation, and a high proliferative index, in addition to poor patient outcome [37]. In submucosal colorectal carcinoma, a positive stromal reaction was associated with lymph node metastasis [31]. However, surprisingly, it was an independent prognostic factor for prolonged disease-free survival in invasive ovarian cancers [38]. Cathepsin K-positive fibroblasts and tumorassociated macrophages were found in aggressive histological types of lung adenocarcinoma (acinar/papillary and mixed type) [13] and in ductal breast carcinoma [10]. All these results, with the addition of those reported in the current study, seem to confirm the important and complex role of the stroma in cancer dissemination. It is well-known that inflammatory cells seen at the invasive margins act either as a defense mechanism against the tumor or, due to various enzymes including cathepsin, can destroy tissue architecture and promote tumor invasion [31]. The expression of cathepsins on the other stromal cells may be involved in the remodeling of stroma within and around the primary tumor, limiting or facilitating its spread. It is also very likely that the promotion of growth reported in some stromal syndecan-1-positive neoplasms is, at least in part, the consequence of storing and presenting heparin-binding growth factors to tumor cells $[5,20]$.

In conclusion, the results of the currant study indicate the association of both epithelial and stromal syndecan-1 and cathepsins $\mathrm{D}$ and $\mathrm{K}$ status with growth and invasion of esophageal squamous cell carcinoma. However, further studies on a larger and more heterogeneous population, especially as far as staging is concerned, are required to elucidate the usefulness of these molecules as markers of progression and prognosis, or even as modes of therapy in patients with the cancer.

\section{References}

[1] Wojciechowska U, Didkowska J, Tarkowski W, Zatonski W. Malignant neoplasms in Poland in 2002. National Register of Tumors. Warsaw: Oncology Center - Maria SklodowskaCurie Institute; 2004

[2] Tew WP, Kelsen DP, Ilson DH. Targeted therapies for esophageal cancer. Oncologist. 2005;10:590-601.

[3] Nair KS, Naidoo R, Chetty R. Expression of cell adhesion molecules in oesophageal carcinoma and its prognostic value. J Clin Pathol. 2005;58:343-351.

[4] Aznavoorian S, Murphy AN, Stetler-Stevenson WG et al. Molecular aspects of tumor cell invasion and metastasis. Cancer. 1993;71:1369-1383.

[ 5] Carey DJ. Syndecans: multifunctional cell - surface co-receptors. Biochem J. 1997;327:1-16.

[6] Beauvais DM, Rapraeger AC. Syndecans in tumor cell adhesion and signaling. Reprod Biol Endocrinol. 2004;2:3-14. 
[7] Nomura T, Katunuma N. Involvement of cathepsins in the invasion, metastasis and proliferation of cancer cells. $J$ Med Invest. 2005;52:1-9.

[ 8] Rochefort H, Liaudet-Coopman E. Cathepsin D in cancer metastasis: a protease and a ligand. APMIS. 1999;107:86-95.

[9] Berdowska I. Cysteine proteases as disease markers. Clin Chim Acta 2004:342:41-69.

[10] Kleer CG, Bloushtain-Qimron N, Chen YH, et al. Epithelial and stromal cathepsin K and CXCL14 expression in breast tumor progression. Clin Cancer Res. 2008;14:5357-5367.

[11] Bühling F, Gerber A, Häckel C et. al. Expression of cathepsin K in lung epithelial cells. Am J Respir Cell Mol Biol. 1999; 20:612-619.

[12] Brubaker KD, Vessella RL, True LD et al. Cathepsin K mRNA and protein expression in prostate cancer progression. J Bone Miner Res. 2003;18:222-230.

[13] Rapa I, Volante M, Cappia S et al. Cathepsin K is selectively expressed in the stroma of lung adenocarcinoma but not in bronchioloalveolar carcinoma. A useful marker of invasive growth. Am J Clin Pathol. 2006;125:847-854.

[14] Hamilton SR, Aaltonen LA. Pathology and genetics of tumors of the digestive system. WHO Classification of Tumors. Lyon: IARC Press; 2000.

[15] Torres CM, Wang HH, Turner JR, et al. Pathologic prognostic factors in squamous cell carcinoma: a follow-up study of 74 patients with or without preoperative chemoradiation therapy. Mod Pathol. 1999;12:961-968.

[16] Anttonen A, Kajanti M, Heikkila P et al. Syndecan-1 expression has prognostic significance in head and neck carcinoma. Br J Cancer. 1999;79:558-564.

[17] Mukunyadzi P, Liu K, Hanna EY et al. Induced expression of syndecan- 1 in the stroma of head and neck squamous cell carcinoma. Mod Pathol. 2003;16:796-801.

[18] Rintala M, Inki P, Klmi P et al. Association of syndecan-1 with tumor grade and histology in primary invasive cervical carcinoma. Gynecol Oncol. 1999;75:372-378.

[19] Chu YQ, Ye ZY, Tao HQ et al. Relationship between cell adhesion molecules expression and the biological behavior of gastric carcinoma. World J Gastroenterol. 2008;4:19901996.

[20] Hashimoto Y, Skacel M, Adams JC. Association of loss of epithelial syndecan-1 with stage and local metastasis of colorectal adenocarcinomas: an immunohistochemical study of clinically annotated tumors. BMC Cancer. 2008;8:185-191.

[21] Li HG, Xie DR, Shen XM et al. Clinicopathological significance of expression of paxillin, syndecan-1 and EMMPRIN in hepatocellular carcinoma. World J Gastroenterol. 2005;14: 1445-1451.

[22] Harada K, Masuda S, Hirano M et al.. Reduced expression of syndecan-1 correlates with histologic dedifferentiation, lymph node metastasis, and poor prognosis in intrahepatic cholangiocarcinoma. Hum Pathol. 2003;34:857-863.

[23] Gökden N, Greene GF, Bayer-Garner IB et al. Expression of CD138 (Syndecan-1) in renal cell carcinoma is reduced with increasing nuclear grade. Appl Immunohistochem Mol Morphol. 2006;14:173-177.

[24] Hasengaowa J, Kodama J, Kusumoto T et al. Prognostic significance of syndecan-1 expression in human endometrial cancer. Ann Oncol. 2005;16:1109-1115.

[25] Mikami S, Ohashi K, Usui Y et al. Loss of syndecan-1 and increased expression of heparanase in invasive esophageal carcinoma. Jpn J Cancer Res. 2001;92:1062-1073.
[26] Day RM, Hao X, Ilyas M et al. Changes in the expression of syndecan-1 in the colorectal adenoma-carcinoma sequence. Virchows Arch. 1999;434:121-125.

[27] Barbareschi M, Maisonneuve P, Aldovini D et al. High syndecan-1 expression in breast carcinoma is related to an aggressive phenotype and to poorer prognosis. Cancer. 2003; 98:474-483.

[28] Shariat SF, Svatek RS, Kabbani W et al. Prognostic value of syndecan-1 expression in patients treated with radical prostatectomy. BJU Int. 2008;101:232-237.

[29] Chen CL, Ou DL. Expression of syndecan-1 (CD138) in nasopharyngeal carcinoma is correlated with advanced stage and poor prognosis. Hum Pathol. 2006;37:1279-1285.

[30] Mylonas I, Makovitzky J, Richter DU et al. Cathepsin D expression in normal, hyperplastic and malignant endometrial tissue: an immunohistochemical analysis. Acta Histochem. 2003; 105:245-252.

[31] Oh-e H, Tanaka S, Kitadai Y et al. Cathepsin D expression as a possible predictor of lymph node metastasis in submucosal colorectal cancer. Eur J Cancer. 2001;37:180-188.

[32] Skrzydlewska E, Sulkowska M, Wincewicz A et al. Evaluation of serum cathepsin B and D in relation to clinicopathological staging of colorectal cancer. World $J$ Gastroenterol. 2005;11:4225-4229.

[33] Cheng AL, Huang WG, Chen ZC et al. Identificating cathepsin D as a biomarker for differentiation and prognosis of nasopharyngeal carcinoma by laser capture microdissection and proteomic analysis. J Proteome Res. 2008;7: 2415-2426.

[34] Kawasaki G, Kato Y, Mizuno A. Cathepsin expression in oral squamous cell carcinoma: relationship with clinicopathologic factors. Oral Surg Oral Med. Oral Pathol Oral Radiol Endod. 2002;93:446-454.

[35] Matsuo K, Kobayashi I, Tsukuba T et al. Immunohistochemical localization of cathepsins D and $\mathrm{E}$ in human gastric cancer: a possible correlation with local invasive and metastatic activities of carcinoma cells. Hum Pathol. 1996;27:184-190.

[36] Merseburger AS, Hennenlotter J, Simon P et al. Cathepsin D expression in renal cell cancer - clinical implications. Eur Urol. 2005;48:519-526.

[37] Ioachim E, Tsanou E, Briasoulis E et al. Clinicopathological study of the expression of hsp27, pS2, cathepsin D and metallothionein in primary invasive breast cancer. Breast. 2003; 12:111-119.

[38] Lösch A, Schindl M, Kohlberger P et al. Cathepsin D in ovarian cancer: prognostic value and correlation with $\mathrm{p} 53$ expression and microvessel density. Gynecol Oncol. 2004;92:545-552.

[39] Ikeguchi M, Sakatani T, Ueta T et al. Correlation between cathepsin D expression and $\mathrm{p} 53$ protein nuclear accumulation in oesophageal squamous cell carcinoma. J Clin Pathol. 2002;55:121-126

[40] Husmann K, Muff R, Bolander ME et al. Cathepsins and osteosarcoma: expression analysis identifies cathepsin $\mathrm{K}$ as an indicator of metastasis. Mol Carcinog. 2008;47;66-73.

[41] Lindeman JHN, Hanemaaijer R, Mulder A et al. Cathepsin K is the principal protease in giant cell tumor of bone. $\mathrm{Am} \mathrm{J}$ Pathol. 2004;165:593-600.

[42] Juuti A, Nordling S, Lundin J et al. Syndecan-1 expression a novel prognostic marker in pancreatic cancer. Oncology 2005;68:97-106.

Submitted:5 August, 2009 Accepted after reviews: 25 November, 2009 